\title{
IncRNA AWPPH promotes the migration and invasion of glioma cells by activating the TGF- $\beta$ pathway
}

\author{
BIN DAI, ZHIYONG XIAO, BEIBEI MAO, GUANGTONG ZHU, HUI HUANG, FENG GUAN, \\ HAIYANG SU, ZHENYANG LIN, WEICHENG PENG and ZHIQIANG HU
}

Department of Neurosurgery, Beijing Shijitan Hospital, Capital Medical University, Beijing 100038, P.R. China

Received July 4, 2018; Accepted May 1, 2019

DOI: 10.3892/ol.2019.10918

\begin{abstract}
The long noncoding RNA (lncRNA) AWPPH, also termed microRNA-4435-2HG, has been associated with the poor prognosis of patients with hepatocellular carcinoma (HCC), and has been demonstrated to promote the progression of HCC and bladder cancer. The present study aimed to investigate the role of IncRNA AWPPH in glioma. The expression levels of AWPPH in tumor tissues obtained from patients with glioma and in plasma samples obtained from patients with glioma and healthy controls were detected by reverse transcription-quantitative polymerase chain reaction. The plasma levels of transforming growth factor (TGF)- $\beta 1$ were measured by an enzyme-linked immunosorbent assay. An AWPPH expression vector was transfected into human glioma cell lines. Subsequently, cancer cell migration and invasion were assessed by Transwell migration and invasion assays, respectively. The expression of TGF- $\beta 1$ in the transfected-glioma cells was detected by western blot analysis. It was identified that AWPPH expression levels in tumor tissues were higher in patients with metastatic glioma; however, no significant differences in AWPPH expression were revealed between patients with different tumor sizes. The plasma levels of AWPPH were positively correlated with the plasma levels of TGF- $\beta 1$ in patients with glioma but not in healthy controls. In addition, AWPPH overexpression enhanced cancer cell migration and invasion, and upregulated TGF- $\beta 1$ expression. Treatment with TGF- $\beta 1$ demonstrated no significant effect on AWPPH expression; however, a TGF- $\beta$ inhibitor attenuated the effects of AWPPH overexpression on cell migration and invasion. Therefore, the present study proposed that AWPPH may promote the migration and invasion of glioma cells by activating the TGF- $\beta$ pathway.
\end{abstract}

Correspondence to: Dr Zhiqiang Hu, Department of Neurosurgery, Beijing Shijitan Hospital, Capital Medical University, 10 Tieyi Road, Yangfangdian, Haidian, Beijing 100038, P.R. China

E-mail: huzhiqiang53000@sina.com

Key words: glioma, long noncoding RNA AWPPH, transforming growth factor- $\beta 1$, migration, invasion

\section{Introduction}

Tumor metastasis poses a key challenge in the treatment of almost all types of human malignancy (1). Once metastasis occurs, surgical resection is an unsuitable treatment strategy, and chemotherapy and radiation therapy can only be administered to prolong the survival of patients but not cure the disease (2). Glioma, a type of cancer, develops from glial cells of the brain or spine and accounts for $>80 \%$ of all cases of malignant brain tumor (3). Different types of glioma may cause different consequences depending on the tumor location (3). Typical clinical symptoms may include seizures, cranial nerve disorders, vomiting, headaches and visual loss (4,5). 'Drop metastases' to the spinal cord frequently occurs in patients with glioma (6). The prevention, early identification and treatment of metastasis are major challenges in overcoming glioma.

Epithelial-mesenchymal transition (EMT) serves pivotal roles in tumor metastasis (7). As a key factor of EMT, transforming growth factor (TGF)- $\beta$ signaling has been reported to serve a role in metastasis of different cancer types (8). Previously, TGF- $\beta$ has been considered as a target for the treatment of numerous types of cancer, including glioma (9). TGF- $\beta$ signaling in certain cases functions via interactions with long noncoding RNAs (lncRNAs), which are a subgroup of noncoding RNAs comprising $>200$ nucleotides and serve critical functions in cancer biology (10). IncRNA AWPPH, a newly identified lncRNA, promotes the progression of hepatocellular carcinoma (11) and bladder cancer (12) via different pathways. The present study identified that AWPPH may promote glioma cell invasion and migration by activating TGF- $\beta$ signaling.

\section{Materials and methods}

Patients. A total of 68 patients with glioma were diagnosed and treated at Beijing Shijitan Hospital (Beijing, China) between March 2014 and January 2018. Among these patients, 48 were included in the present study according to the inclusion and exclusion criteria. The inclusion criteria were as follows: i) Patient diagnosed pathologically using tumor biopsies; ii) patient treated for the first time; and iii) patient willing to participate. The exclusion criteria were as follows: i) Patient diagnosed with another type of severe disease; ii) patient received treatment prior to admission; and iii) patient 
$>70$ years. The 48 patients included 28 males and 20 females with a mean age of 47.2 \pm 7.1 years (range, 27-68 years). In addition, a total of 38 healthy controls were included in the physical health examination center of the same hospital during the same time period to match the age and sex distributions of patients. The control group included 22 males and 16 females with a mean age of 46.2 \pm 5.1 years (range, 25-69 years). No significant differences in age or sex were identified between the two groups. The Ethics Committee of Beijing Shijitan Hospital (Beijing, China) approved the present study and all participants provided written informed consent.

Specimen collection. Tumor tissue samples (100-150 mg) were collected from patients with glioma during tumor biopsies for pathological examination. Blood was extracted from the elbow vein of patients and healthy controls on the day of admission. Blood was kept in a BD Vacutainer ${ }^{\circledR}$ Plasma Preparation Tube for $30 \mathrm{~min}$, followed by centrifugation at 1,600 x $\mathrm{g}$ at room temperature for $20 \mathrm{~min}$ to collect plasma.

Reverse transcription-quantitative polymerase chain reaction $(R T-q P C R)$. Total RNA was extracted from tissues and in vitro cultivated cells using TRIzol ${ }^{\circledR}$ reagent (Invitrogen; Thermo Fisher Scientific, Inc., Waltham, MA, USA). In cases of TGF- $\beta 1$ treatment, cells were first incubated with 5 or $10 \mathrm{ng} / \mathrm{ml}$ TGF- $\beta 1$ (Sigma-Aldrich; Merck KGaA) for $24 \mathrm{~h}$ at $37^{\circ} \mathrm{C}$ prior to RNA extractions. Total RNA was transcribed into complementary DNA (cDNA) by RT using the SuperScript III Reverse Transcriptase kit (Thermo Fisher Scientific, Inc.). The reaction conditions were as follows: $50^{\circ} \mathrm{C}$ for $20 \mathrm{~min}$ and $80^{\circ} \mathrm{C}$ for 5 min. SYBR ${ }^{\circledR}$ Green Real-Time PCR Master mix (Thermo Fisher Scientific., Inc.) was used to prepare PCR reactions. The following primers were used in the PCR reactions: Human lncRNA AWPPH forward, 5'-CTGGATGGTCGCTGCTTT TTA-3' and reverse, 5'-AGGGGGATGAGTCGTGATTT-3'; and $\beta$-actin forward, 5'-GACCTCTATGCCAACACAGT-3' and reverse, 5'-AGTACTTGCGCTCAGGAGGA-3'. The PCR reaction conditions were as follows: $45 \mathrm{sec}$ at $95^{\circ} \mathrm{C}$, followed by 40 cycles of $12 \mathrm{sec}$ at $95^{\circ} \mathrm{C}$ and $38 \mathrm{sec}$ at $56^{\circ} \mathrm{C}$. PCR reactions were performed on CFX96 Touch $^{\text {TM }}$ Real-Time PCR Detection System (Bio-Rad). Data were evaluated using the $2^{-\Delta \Delta \mathrm{Cq}}$ method (13) to normalize the AWPPH expression level to the endogenous control $\beta$-actin.

Enzyme-linked immunosorbent assay (ELISA). Plasma levels of TGF- $\beta 1$ were measured using an human TGF- $\beta 1$ ELISA kit (cat. no. DY240, R\&D Systems, Inc., Minneapolis, MN, USA), according to the manufacturer's protocol. Plasma levels of TGF- $\beta 1$ were normalized for platelet activation using PF4 ELISA using a human PF4 ELISA Kit (ab100628, Abcam).

Cell culture and transfection. Two human glioma cell lines CCD-25Lu and Hs 683 were purchased from the American Type Culture Collection (ATCC; Manassas, VA, USA). Cells were cultured in ATCC-formulated Eagle's Minimum Essential medium (cat. no. 30-2003; ATCC) supplemented with $10 \%$ fetal bovine serum (FBS, ATCC). AWPPH cDNA was purchased from Sangon (Shanghai, China) and inserted into the EcoR1-linearized pIRSE2 vector (Clontech Laboratories, Inc., Mountainview, CA, USA). AWPPH cDNA cloned using aforementioned RNA samples. Following the same reverse transcriptions, PCR amplification was performed using Phusion ${ }^{\circledR}$ High-Fidelity DNA Polymerase (NEB, USA) through following thermal conditions: $1 \mathrm{~min}$ at $95^{\circ} \mathrm{C}$, followed by 30 cycles of $12 \mathrm{sec}$ at $95^{\circ} \mathrm{C}, 10 \mathrm{sec}$ at $55^{\circ} \mathrm{C}$ and $2 \mathrm{~min}$ at $72^{\circ} \mathrm{C}$. Cloning primer sequences were as follows: AWPPH forward, 5'-ACAAGTATGAAGAGAATGTCGG-3' and reverse, 5'-AGA TTTTTCCCACATGTGATTTTATTTC-3'. Lipofectamine ${ }^{\circledR}$ 2000 reagent (cat. no. 11668-019; Invitrogen; Thermo Fisher Scientific, Inc.) was first mixed with $10 \mathrm{nM}$ vector (empty vector was a negative control, NC) and kept at room temperature for $30 \mathrm{~min}$ to allow the formation of reagent-vector complexes. Subsequently, the complexes were mixed with CCD-25Lu and Hs 683 cells and kept at $37^{\circ} \mathrm{C}$ for $6 \mathrm{~h}$ to allow transfection. The media was then immediately replaced with fresh cell culture medium to maintain cells. Overexpression of AWPPH was confirmed by RT-qPCR prior to subsequent experimentation.

In vitro cell migration and invasion assays. In vitro cell migration and invasion abilities of CCD-25Lu and Hs 683 cells were detected by Transwell migration and invasion assays. In cases of TGF- $\beta$ inhibitor treatment, cells were incubated with 100 nM TGF- $\beta$ inhibitor LY2109761 (Sigma-Aldrich; Merck $\mathrm{KGaA}$ ) for $24 \mathrm{~h}$ at $37^{\circ} \mathrm{C}$ prior to use. For the migration assay, $0.1 \mathrm{ml} 1 \%$ cell suspension (1\% FBS, ATCC) containing $3 \times 10^{3}$ cells was added to the upper Transwell chamber and RPMI-1640 medium (Thermo Fisher Scientific, Inc.) containing $20 \%$ FBS (ATCC) was added to the lower chamber. Cells were incubated at room temperature for $2 \mathrm{~h}$. The membranes were then washed and stained with $0.5 \%$ crystal violet (Sigma-Aldrich; Merck KGaA, Darmstadt, Germany) for $15 \mathrm{~min}$ at room temperature. Cells were counted under an light microscope (40x). Five visual fields were selected from each membrane. For the invasion assay, the upper Transwell chamber was pre-coated with Matrigel (cat. no. 356234; EMD Millipore, Billerica, MA, USA).

Western blot analysis. Radioimmunoprecipitation assay lysis solution (Thermo Fisher Scientific, Inc.) was used for protein extraction from CCD-25Lu and $\mathrm{Hs} 683$ cells and protein concentrations were measured by BSA assay. Subsequently, $10 \%$ SDS-PAGE was performed with $25 \mu \mathrm{g}$ denatured protein in each well. Following protein transfer to polyvinylidene difluoride membranes, blocking was performed with $5 \%$ skimmed milk at room temperature for $2 \mathrm{~h}$. After three washes with TBS and $0.3 \%$ Tween (TBST) buffer, the membranes were incubated with rabbit anti-human primary antibodies against TGF- $\beta 1$ (1:1,500; cat. no. ab92486; Abcam, Cambridge, UK) and GAPDH (1:1,200; cat. no. ab9485; Abcam) at $4^{\circ} \mathrm{C}$ overnight. The membranes were then washed three times with TBST buffer, followed by incubation with goat anti-rabbit IgG-horseradish peroxidase secondary antibody $(1: 1,000$; cat. no. MBS435036; MyBioSource, Inc., San Diego, CA, USA) for $2 \mathrm{~h}$ at room temperature. Subsequently, the membranes were washed three times with TBST buffer and the Pierce ECL Western Blotting Substrate (Thermo Fisher Scientific, Inc.) was used to develop signals. Signals were scanned using MYECL ${ }^{\mathrm{TM}}$ Imager (Thermo Fisher Scientific, Inc.). TGF- $\beta 1$ expression was normalized to that of GAPDH using ImageJ 1.6 software (National Institutes of Health, Bethesda, MD, USA). 

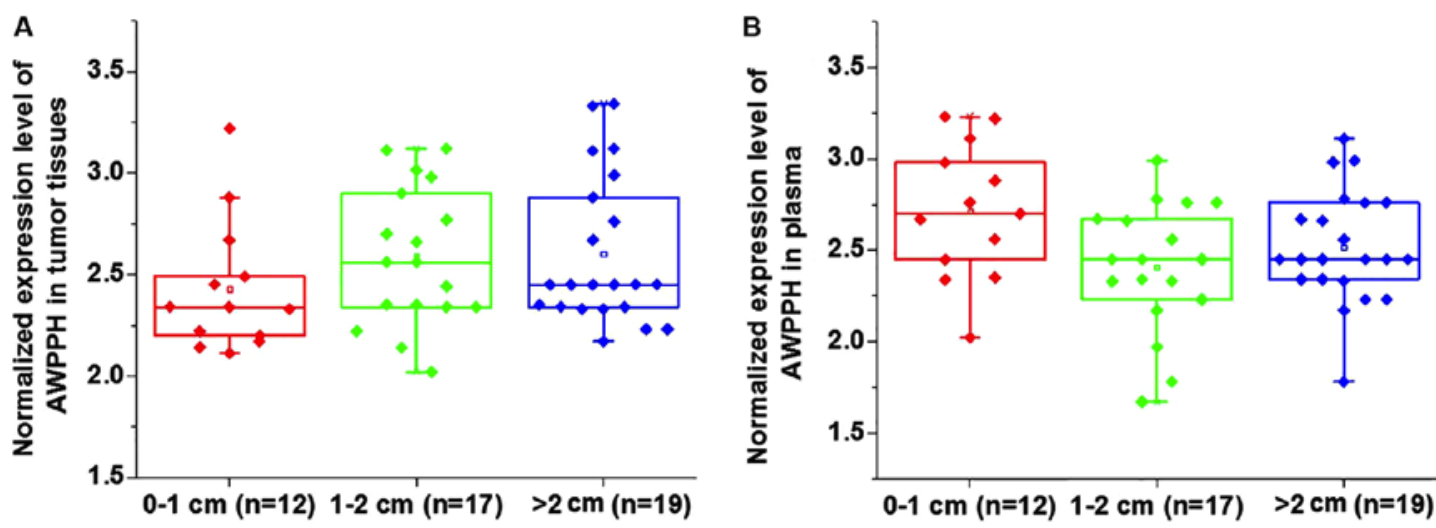

Figure 1. Comparison of AWPPH expression levels in patients with different tumor sizes. AWPPH expression levels in (A) tumor tissues and (B) plasma obtained from patients with different tumor sizes. No significant differences in the expression levels of AWPPH in tumor tissues and plasma were identified among patients with different tumor sizes $(\mathrm{P}>0.05)$.

Statistical analysis. GraphPad Prism 6 (GraphPad Software, Inc., La Jolla, CA, USA) was used for all statistical analysis. Data of 3 biological replicates are presented as the mean \pm standard deviation. A Student's t-test was used for comparisons between two groups and one-way analysis of variance followed by Tukey's test was used for comparisons among multiple groups. Pearson's correlation coefficient was used for correlation analysis. $\mathrm{P}<0.05$ was considered to indicate a statistically significant difference.

\section{Results}

Comparison of AWPPH expression in patients with different tumor sizes. Based on the diameter of primary tumors, 12 patients presented with a tumor diameter between $0-1 \mathrm{~cm}$, 17 patients had a tumor diameter between $1-2 \mathrm{~cm}$ and 19 patients presented with a tumor diameter $>2 \mathrm{~cm}$. No significant differences in the expression of AWPPH were identified in tumor tissues (Fig. 1A) and the plasma (Fig. 1B) among patients with different tumor sizes $(\mathrm{P}>0.05)$.

Comparison of AWPPH expression in patients with or without distant tumor metastasis. Distant tumor metastasis was observed in 22 patients. As presented in Fig. 2, the expression levels of AWPPH in tumor tissues (Fig. 2A) and plasma (Fig. 2B) were significantly higher in patients with metastatic glioma compared with patients with non-metastatic glioma $(\mathrm{P}<0.05)$. In addition, the plasma levels of AWPPH were significantly increased in patients with metastatic glioma compared with the controls and non-metastatic patients, while no significant differences in the plasma levels of AWPPH were identified between the non-metastatic glioma and control groups ( $\mathrm{P}<0.05$; Fig. 2B).

Correlation between plasma levels of AWPPH and TGF- $\beta 1$ in patients with glioma and healthy controls. The current data suggest that AWPPH is likely involved in the regulation of tumor metastasis but not tumor growth in glioma. TGF- $\beta$ signaling serves pivotal roles in the metastasis of different types of cancer, including glioma (14). Therefore, the correlation between the plasma levels of AWPPH and TGF- $\beta 1$ was analyzed using all plasma samples. As presented in Fig. 3, the plasma levels of AWPPH were positively correlated with the plasma levels of TGF- $\beta 1$ in patients with glioma $(\mathrm{P}<0.0001$; Fig. 3A) but not in healthy controls ( $\mathrm{P}=0.5927$; Fig. 3B).

AWPPH overexpression promotes the expression of TGF- $\beta 1$ in glioma cells. To further investigate the association between AWPPH and TGF- $\beta 1$ in glioma, an AWPPH expression vector was transfected into the human glioma cell lines CCD-25Lu and Hs 683 (P<0.05; Fig. 4A), and the expression of TGF- $\beta 1$ was detected by western blot analysis $(\mathrm{P}<0.05$; Fig. 4B). The results revealed that compared with control group and negative control group, AWPPH overexpression significantly promoted the expression of TGF- $\beta 1$ in CCD-25Lu and Hs 683 cells $(\mathrm{P}<0.05$; Fig. 4B). By contrast, treatment with 5 or $10 \mathrm{ng} / \mathrm{ml}$ TGF- $\beta 1$ (Sigma-Aldrich; Merck KGaA) demonstrated no significant effects on AWPPH expression in CCD-25Lu and Hs 683 cells ( $\mathrm{P}>0.05$; Fig. 4C).

AWPPH overexpression promotes the migration and invasion of glioma cells. The present data suggest that AWPPH is likely involved in the metastasis of glioma. Therefore, Transwell migration and invasion assays were performed to investigate the effects of AWPPH on the migration and invasion of in vitro cultured cells. As presented in Fig. 5, compared with the control (C) group, AWPPH overexpression significantly enhanced the migration (Fig. 5A) and invasion (Fig. 5B) of the human glioma cell lines CCD-25Lu and Hs 683. In addition, treatment with 100 nM TGF- $\beta$ inhibitor LY2109761 (Sigma-Aldrich; Merck $\mathrm{KGaA}$ ) significantly inhibited cell migration and invasion compared with the control, and significantly attenuated the effects of AWPPH overexpression on the migration (Fig. 5A) and invasion (Fig. 5B) of glioma cells.

\section{Discussion}

It is understood that the IncRNA AWPPH, a newly identified lncRNA, promotes the progression of hepatocellular carcinoma (11) and bladder cancer (12) via interactions with different signaling molecules. The key finding of the present study is that AWPPH may serve an oncogenic role in glioma. The molecular mechanism of AWPPH is likely associated with the activation of TGF- $\beta$ signaling. 

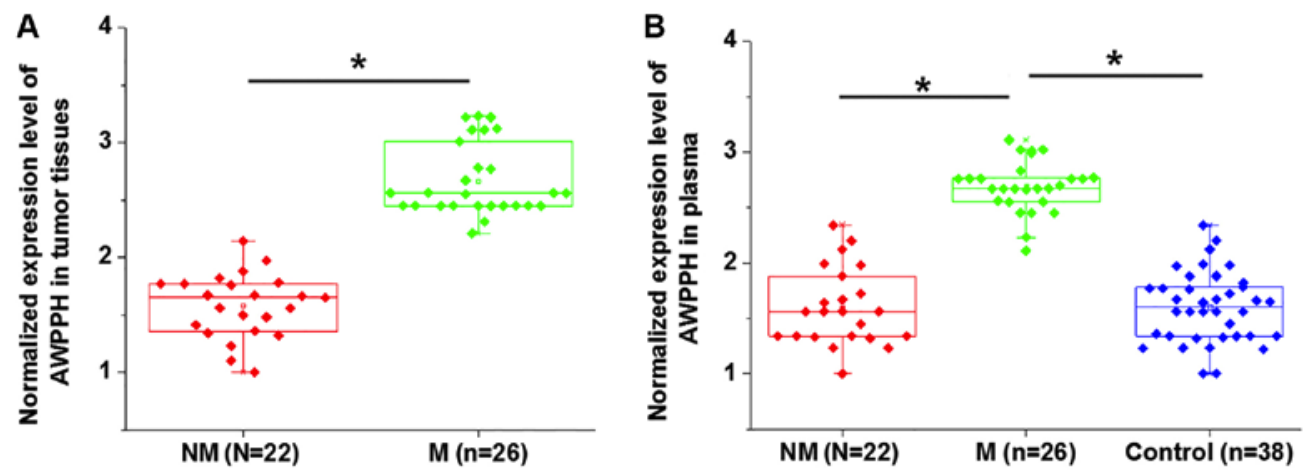

Figure 2. Comparison of AWPPH expression levels in patients with or without distant tumor metastasis. AWPPH expression levels in (A) tumor tissues and (B) plasma obtained from patients with or without distant tumor metastasis. Expression levels of AWPPH in tumor tissues and plasma were significantly higher in patients with M compared with patients with NM. Plasma levels of AWPPH were also significantly higher in patients with M compared with the controls, while no significant differences in plasma levels of AWPPH were identified between patients with NM and the control group. "P<0.05. NM, non-metastatic glioma; M, metastatic glioma.
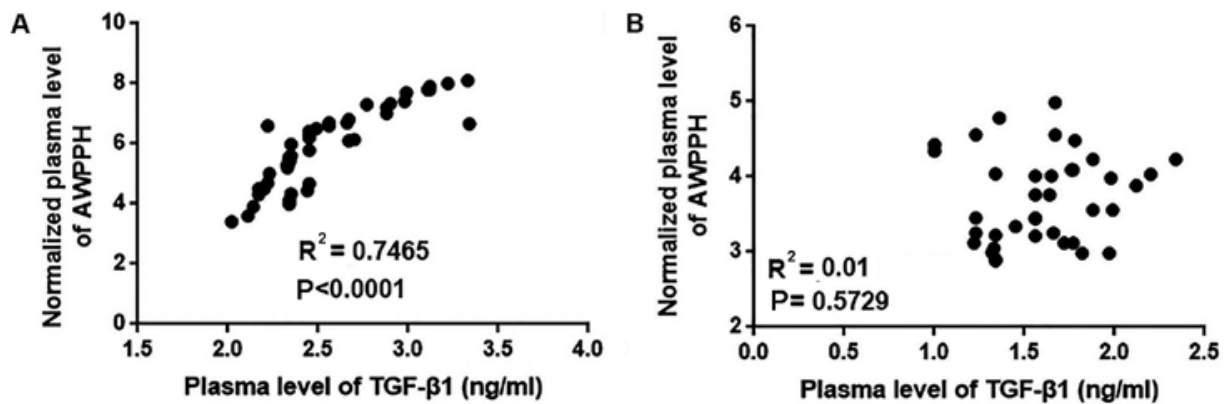

Figure 3. Correlation between plasma levels of AWPPH and TGF- $\beta 1$ in patients with glioma and healthy controls. The correlation between plasma levels of AWPPH and TGF- $\beta 1$ in (A) patients with glioma and (B) healthy controls. Plasma levels of AWPPH were positively correlated with plasma levels of TGF- $\beta 1$ in patients with glioma but not in healthy controls. TGF- $\beta 1$, transforming growth factor- $\beta 1$.

A

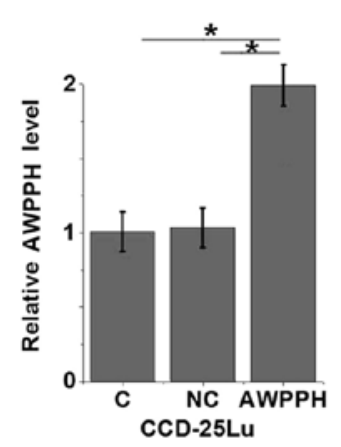

C

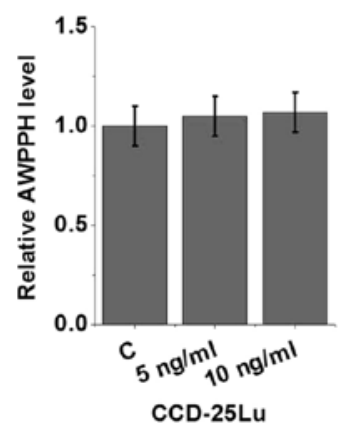

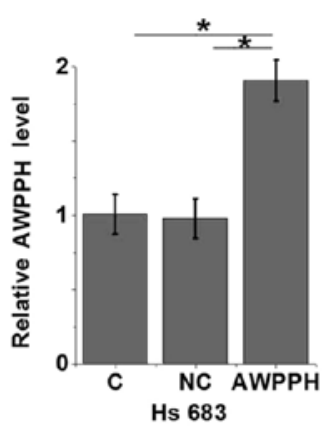

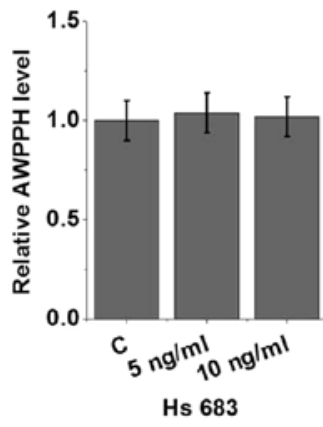

CCD-25Lu

C NC AWPPH
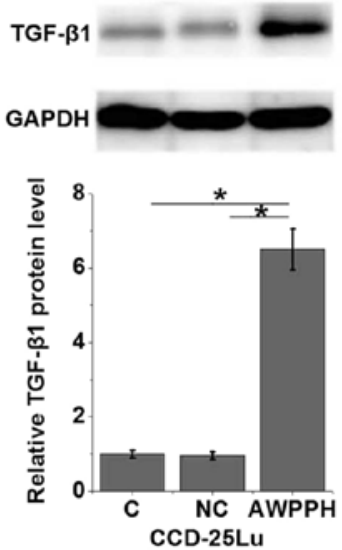

GAPDH

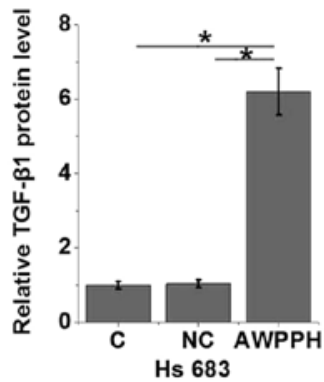

Figure 4. AWPPH overexpression promotes the expression of TGF- $\beta 1$ in glioma cells. (A) Confirmation of AWPPH overexpression by qPCR. (B) The effects of AWPPH overexpression on TGF- $\beta 1$ expression. (C) The effects of TGF- $\beta 1$-treatment on AWPPH expression. AWPPH overexpression significantly promoted the expression of TGF- $\beta 1$ in CCD-25Lu and Hs 683 cells. By contrast, treatment with 5 and $10 \mathrm{ng} / \mathrm{ml} \mathrm{TGF-} \beta 1$ demonstrated no significant effects on AWPPH expression in glioma cells. ${ }^{*} \mathrm{P}<0.05$. TGF- $\beta 1$, transforming growth factor- $\beta 1$; $\mathrm{NC}$, negative control; $\mathrm{C}$, control. 

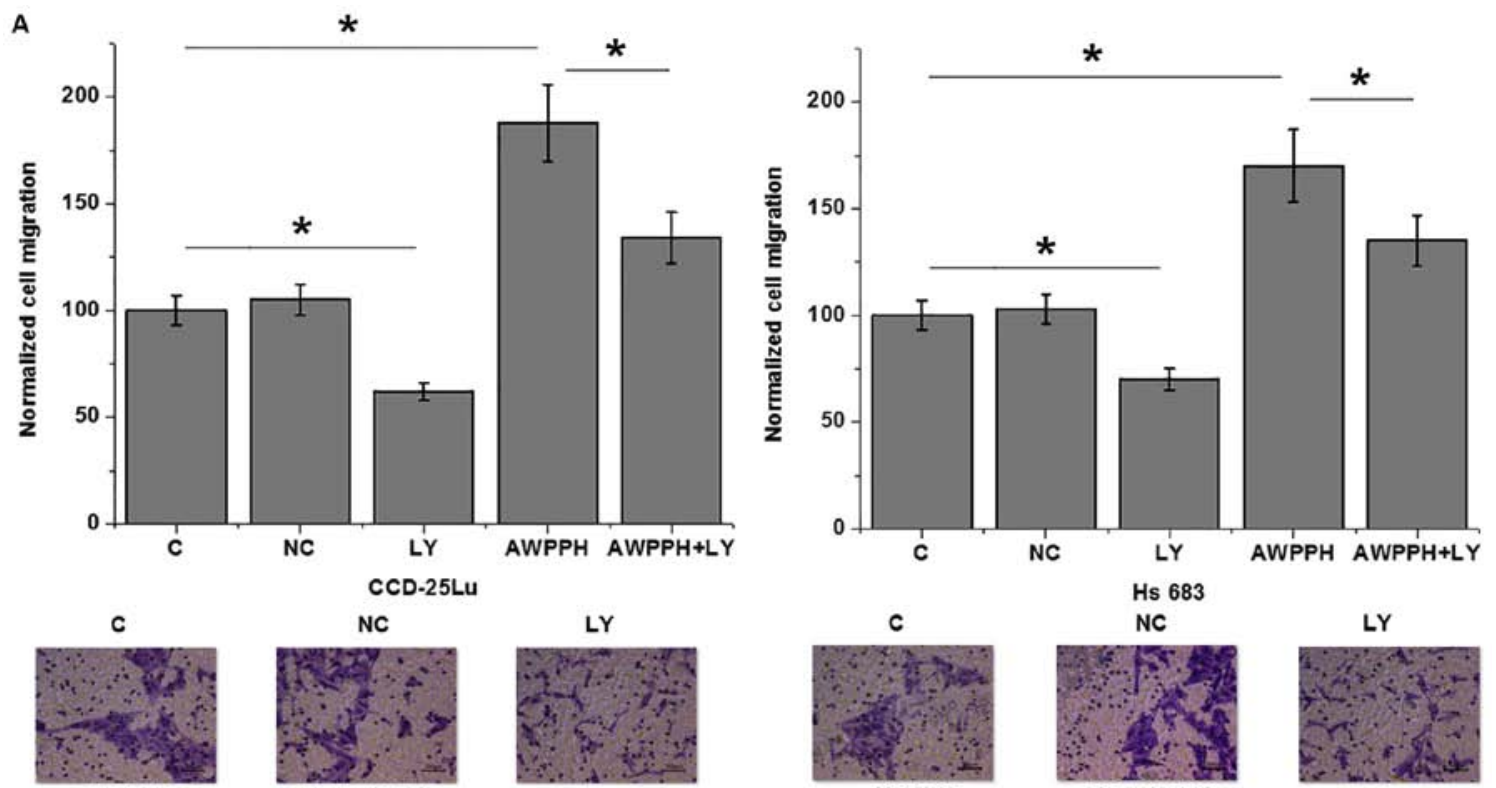

NC
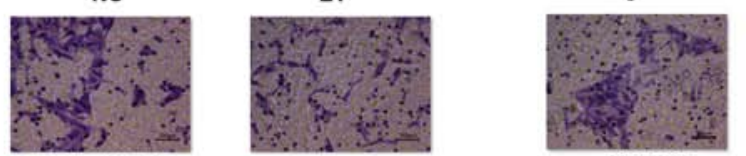

NC

AWPPH
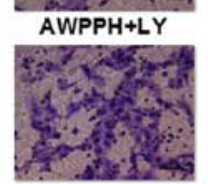

AWPPH
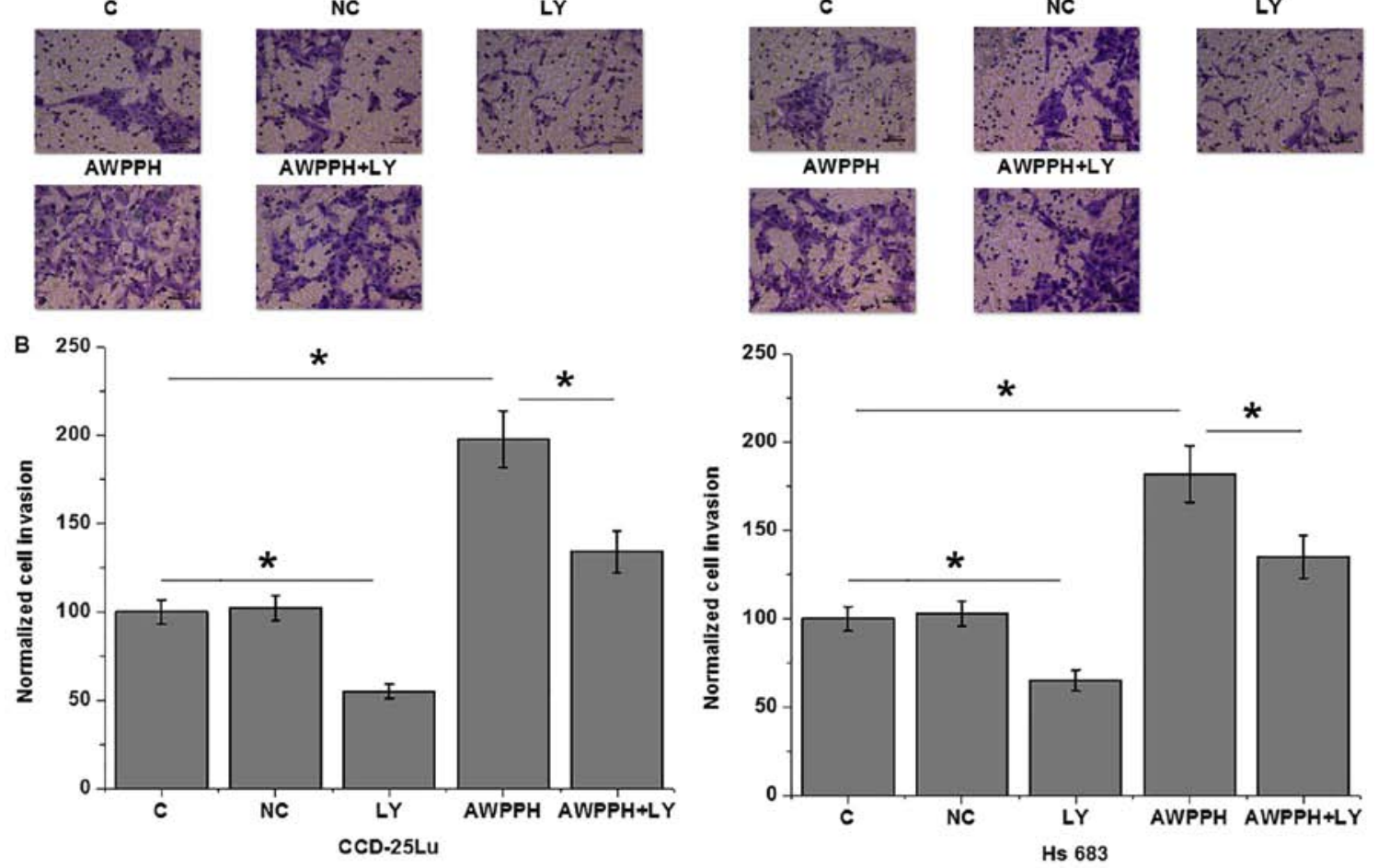

c

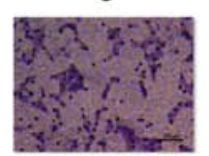

AWPPH

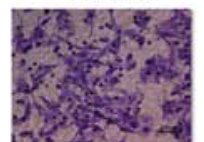

NC

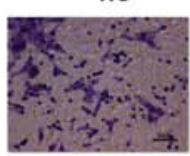

AWPPH+LY

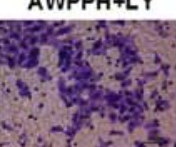

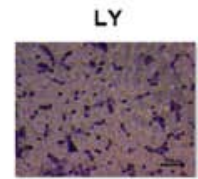

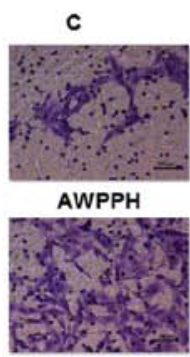

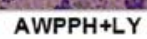

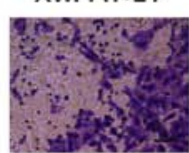

NC

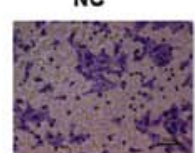

AWPPH+LY

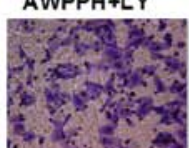

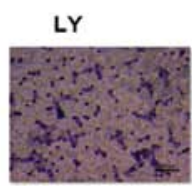

Figure 5. AWPPH overexpression promotes the migration and invasion of glioma cells. The effects of AWPPH overexpression and the TGF- $\beta$ inhibitor LY on the (A) migration and (B) invasion of human glioma cell lines CCD-25Lu and Hs 683. AWPPH overexpression significantly enhanced the migration and invasion of CCD-25Lu and Hs 683 cells. In addition, treatment with $100 \mathrm{nM}$ TGF- $\beta$ inhibitor LY significantly inhibited cell migration and invasion, and significantly attenuated the effects of AWPPH overexpression on migration and invasion. Images were obtained under 40x magnification. * $<0.05$. LY, LY2109761; $\mathrm{C}$, control; NC, negative control.

In a study investigating hepatocellular carcinoma, Zhao et al (11) identified AWPPH as a novel lncRNA that is significantly upregulated in cancer tissues compared with healthy liver tissues. In another study, significantly upregulated expression levels of AWPPH were observed in bladder cancer tissues and cells compared with normal bladder tissues and cells, which indicates a potential oncogenic role of AWPPH in bladder cancer. In contrast to the aforementioned findings, the present study investigated the differential expression of AWPPH in patients with different tumor sizes and patients with or without distant tumor metastasis. This strategy was selected as the expression levels of AWPPH were not significantly different in numerous patients with glioma compared with the healthy controls. In addition, a simple comparison between healthy 
controls and patients with glioma is insufficient to reveal the role of AWPPH in a specific process of tumor progression, including tumor growth and metastasis. The present study revealed that AWPPH is not differentially expressed in patients with different tumor sizes. However, patients with metastatic glioma exhibited significantly higher AWPPH expression levels compared with patients with non-metastatic glioma. This suggests that AWPPH is involved in the metastasis of glioma.

TGF- $\beta$ signaling inhibits tumor growth at the initial stage of cancer development but promotes tumor metastasis via EMT at later stages (15). Differential expression of TGF- $\beta$ isoforms has also been observed in different stages of glioma (16). It has been reported that TGF- $\beta$ may serve as a potential target for the treatment of glioma (14). The cross talk between TGF- $\beta$ and lncRNAs has been extensively reported in different types of malignancy (17-19). However, to the best of our knowledge, studies regarding the interactions between TGF- $\beta$ and lncRNAs are relatively rare. The present study identified a significant positive correlation between the plasma levels of TGF- $\beta 1$ and AWPPH in patients with glioma. In addition, in vitro experiments demonstrated that AWPPH may be an upstream activator of TGF- $\beta 1$ in glioma. AWPPH overexpression upregulated TGF- $\beta 1$ expression in human glioma cells, while TGF- $\beta 1$ treatment demonstrated no significant effects on AWPPH expression. Additionally, treatment with the TGF- $\beta$ inhibitor LY2109761 significantly inhibited cell migration and invasion, and attenuated the effects of AWPPH overexpression. These data also suggest that inhibition of endogenous TGF- $\beta$ may serve as a therapeutic target for glioma.

No significant correlation between plasma TGF- $\beta 1$ and AWPPH levels was observed in healthy controls. Therefore, the present study did not include a normal human brain tissue cell line as a control. This also suggests that the regulatory role of AWPPH on TGF- $\beta 1$ is glioma-specific or specific to disease conditions. Notably, LINC00152, a homolog of AWPPH, promotes the invasion of glioblastoma via a 3'-hairpin structure, which serves as a protein-binding site (20). This is consistent with the results of the present study.

In conclusion, it was identified that AWPPH is upregulated in glioma. Overexpression of AWPPH may promote the migration and invasion of glioma cells by activating TGF- $\beta$ signaling. However, the present study is limited by a small sample size. Studies with larger sample sizes are required to further validate the current conclusions. In addition, we only demonstrated possible AWPPH-TGF- $\beta 1$ sequential signaling in glioma, whether this interaction occurs in a direct or indirect manner requires further investigation.

\section{Acknowlegements}

Not applicable.

\section{Funding}

No funding was received.

\section{Availability of data and materials}

The datasets used and/or analyzed during the current study are available from the corresponding author on reasonable request

\section{Authors' contributions}

$\mathrm{BD}, \mathrm{ZX}$ and $\mathrm{ZH}$ conceived and designed the study. BD, ZX, BM, GZ, HH, FG, HS, ZL, WP performed the experiments. BD, ZX, GZ, HH, FG, HS, ZL and WP wrote the paper. BD, $\mathrm{ZX}, \mathrm{BM}, \mathrm{ZX}, \mathrm{BM}$ and $\mathrm{GZ}$ reviewed and edited the manuscript. All authors read and approved the manuscript.

\section{Ethics approval and consent to partcipate}

The Ethics Committee of Beijing Shijitan Hospital (Beijing, China)approved the present study and all participants provided written informed consent.

\section{Patient consent for publication}

All individuals involved in this study provided written informed consent.

\section{Competing interests}

The authors declare that they have no competing interests.

\section{References}

1. Valastyan S and Weinberg RA: Tumor metastasis: Molecular insights and evolving paradigms. Cell 147: 275-292, 2011.

2. Steeg PS: Tumor metastasis: Mechanistic insights and clinical challenges. Nat Med 12: 895-904, 2006

3. Goodenberger ML and Jenkins RB: Genetics of adult glioma. Cancer Genet 205: 613-621, 2012.

4. Omuro A and DeAngelis LM: Glioblastoma and other malignant gliomas: A clinical review. JAMA 310: 1842-1850, 2013.

5. Mishra MV, Andrews DW, Glass J, Evans JJ, Dicker AP, Shen X and Lawrence YR: Characterization and outcomes of optic nerve gliomas: A population-based analysis. J Neurooncol 107: 591-597, 2012.

6. Stark AM, van de Bergh J, Hedderich J, Mehdorn HM and Nabavi A: Glioblastoma: Clinical characteristics, prognostic factors and survival in 492 patients. Clin Neurol Neurosurg 114: 840-845, 2012

7. Heerboth S, Housman G, Leary M, Longacre M, Byler S, Lapinska K, Willbanks A and Sarkar S: EMT and tumor metastasis. Clin Transl Med 4: 6, 2015.

8. Katsuno Y, Lamouille S and Derynck R: TGF- $\beta$ signaling and epithelial-mesenchymal transition in cancer progression. Curr Opin Oncol 25: 76-84, 2013.

9. Joseph JV, Balasubramaniyan V, Walenkamp A and Kruyt FA: TGF- $\beta$ as a therapeutic target in high grade gliomas-promises and challenges. Biochem Pharmacol 85: 478-485, 2013.

10. Gutschner T and Diederichs S: The hallmarks of cancer: A long non-coding RNA point of view. RNA Biol 9: 703-719, 2012.

11. Zhao X, Liu Y and Yu S: Long noncoding RNA AWPPH promotes hepatocellular carcinoma progression through YBX1 and serves as a prognostic biomarker. Biochim Biophys Acta Mol Basis Dis 1863: 1805-1816, 2017.

12. Zhu F, Zhang X, Yu Q, Han G, Diao F, Wu C and Zhang Y: LncRNA AWPPH inhibits SMAD4 via EZH2 to regulate bladder cancer progression. J Cell Biochem 119: 4496-4505, 2018.

13. Livak KJ and Schmittgen TD: Analysis of relative gene expression data using real-time quantitative PCR and the 2(-Delta Delta C(T)) method. Methods 25: 402-408, 2001.

14. Han J, Alvarez-Breckenridge CA, Wang QE and Yu J: TGF- $\beta$ signaling and its targeting for glioma treatment. Am J Cancer Res 5: 945-955, 2015.

15. Mirzaei $\mathrm{H}$ and Faghihloo E: Viruses as key modulators of the TGF- $\beta$ pathway; a double-edged sword involved in cancer. Rev Med Virol 28, 2018 doi: 10.1002/rmv.1967.

16. Kjellman C, Olofsson SP, Hansson O, Von Schantz T, Lindvall M, Nilsson I, Salford LG, Sjögren HO and Widegren B: Expression of TGF-beta isoforms, TGF-beta receptors, and SMAD molecules at different stages of human glioma. Int J Cancer 89: 251-258, 2000. 
17. Li C, Wan L, Liu Z, Xu G, Wang S, Su Z, Zhang Y, Zhang C, Liu $X$, Lei Z and Zhang HT: Long non-coding RNA XIST promotes TGF- $\beta$-induced epithelial-mesenchymal transition by regulating miR-367/141-ZEB2 axis in non-small-cell lung cancer. Cancer Lett 418: 185-195, 2018.

18. Li Z, Dong M, Fan D, Hou P, Li H, Liu L, Lin C, Liu J, Su L, Wu L, et al: LncRNA ANCR down-regulation promotes TGF- $\beta$-induced EMT and metastasis in breast cancer. Oncotarget 8: 67329-67343, 2017.

19. Zhao JJ, Hao S, Wang LL, Hu CY, Zhang S, Guo LJ, Zhang G, Gao B, Jiang Y, Tian WG and Luo DL: Long non-coding RNA ANRIL promotes the invasion and metastasis of thyroid cancer cells through TGF- $\beta /$ Smad signaling pathway. Oncotarget 7 : 57903-57918, 2016.
20. Reon BJ, Takao Real Karia B, Kiran M and Dutta A: LINC00152 promotes invasion through a 3'-Hairpin structure and associates with prognosis in glioblastoma. Mol Cancer Res 16: 1470-1482, 2018.

c) (i) $(-)$ This work is licensed under a Creative Commons EY NC ND Attribution-NonCommercial-NoDerivatives 4.0 International (CC BY-NC-ND 4.0) License. 